\title{
Aflibercept for clinically significant diabetic macular edema: 12-month results in daily clinical practice
}

This article was published in the following Dove Press journal:

Clinical Ophthalmology

\author{
Rafael Campos Polo \\ Consuelo Rubio Sánchez \\ Diego Manuel García \\ Guisado \\ María José Díaz Luque \\ Unit of Retina, Department of \\ Ophthalmology, Hospital Virgen del \\ Puerto, Plasencia, Cáceres, Spain
}

Purpose: To assess the effectiveness and safety of intravitreal aflibercept in clinically significant diabetic macular edema (DME) in daily clinical practice.

Methods: Prospective, open-label, single-center study. Anti-vascular endothelial growth factor naïve patients with clinically significant DME received intravitreal injections of aflibercept $2 \mathrm{mg}$, five monthly doses followed by a fixed schedule every 2 months for 12 months. The mean change in best-corrected visual acuity (BCVA) (Early Treatment Diabetic Retinopathy Study [ETDRS] letters) was the primary outcome.

Results: The mean BCVA improved significantly as compared with baseline at 12 months of treatment (47.3 [14.2] vs 62.2 [13.9] ETDRS letters, $P<0.001$ ). Significant improvement in BCVA was already observed at visit 2 after the loading doses of aflibercept. At 12 months, gains in ETDRS letters were documented in all eyes (100\%), with gains $\geq 10$ letters in $89.6 \%, \geq 15$ letters in $65.5 \%$, and $\geq 20$ letters in $6.9 \%(\mathrm{n}=2)$. A significant reduction in central macular thickness from a mean of $460.5(11.8) \mu \mathrm{m}$ at baseline to $229.0(43.8) \mu \mathrm{m}$ at 12 months $(P<0.001)$ was observed. Significant reductions of central macular thickness were already observed after the loading doses and continued lowering throughout the study period. No adverse events occurred.

Conclusion: Aflibercept as a first-line therapy was effective and well tolerated for treating clinically significant DME in naïve patients in daily practice. Successful results in terms of improvement of visual and reduction in central macular thickness contribute to provide evidence for the positioning of aflibercept as a first-line indication of newly diagnosed clinically significant DME.

Keywords: aflibercept, central macular thickness, diabetic macular edema, routine clinical practice, visual acuity

\section{Introduction}

Diabetic macular edema (DME) is a major cause of vision loss associated with diabetic retinopathy and is characterized by exudation and accumulation of extracellular fluid in the macula secondary to an increase in vascular permeability. ${ }^{1,2}$ A review conducted in 2012 suggested that up to $7 \%$ of people with diabetes may have DME. ${ }^{3}$ Among population-based studies, the prevalence of DME in patients with type 1 diabetes was between $4.2 \%$ and $7.9 \%$. In patients with type 2 diabetes, it was between $1.4 \%$ and $12.8 \% .{ }^{4}$ However, the global prevalence of DME is likely to increase along with the increasing prevalence of diabetes mellitus, particularly in view of the current epidemics of type 2 diabetes. $^{5}$

Although the pathogenesis of DME is multifactorial, including capillary endothelial vascular dysfunction, local inflammatory activity, cellular hypoxia, oxidative stress,
Correspondence: Rafael Campos Polo Department of Ophthalmology, Hospital Virgen del Puerto, Paraje Valcorchero s/n, E- 10600 Plasencia, Cáceres, Spain

$\mathrm{Tel}+34927428300$

Fax +34927428324

Email rafacampospolo@hotmail.com 
breakdown of the blood-retinal barrier, and retinal neurodegeneration, the overexpression of vascular endothelial growth factor (VEGF) has been identified as a key contributor to the development of DME. ${ }^{6,7}$ The proteins VEGF-A, VEGF-B, and the placental growth factor (PIGF) are members of the VEGF family and the most relevant for ocular disease. These proteins after binding to specific receptors (VEGFR-1 and VEGFR-2) initiate a cascade of events leading to vasculogenesis and angiogenesis. ${ }^{8,9}$ Awareness of the role of VEGF and inflammatory mediators in the pathogenesis of DME has prompted the development and widespread use of anti-VEGF inhibitors that can target these pathways. ${ }^{10-12}$ Anti-VEGF treatment has superseded macular laser treatment and is now the first-line therapy for DME involving the central macula.

The monoclonal antibodies bevacizumab ${ }^{13,14}$ and ranibizumab ${ }^{15,16}$ target VEGF-A isoforms, whereas aflibercept, unlike the monoclonal antibodies, is a soluble decoy receptor fusion protein that inhibits PIGF in addition to all isoforms of VEGF-A and VEGF-B. ${ }^{17-19}$ Also, aflibercept has a longer duration of action than other anti-VEGF agents and has a 100-fold greater binding affinity for VEGF-A than intravitreal ranibizumab, ${ }^{20-22}$ which confers the advantage of the potential for less frequent dosing of aflibercept, with substantial saving in cost and treatment burden to patients. ${ }^{23}$ The efficacy and safety of aflibercept in diabetic patients with DME were demonstrated in the landmark Phase III VISTADME and VIVID-DME studies. ${ }^{24,25}$ The administration of $2 \mathrm{mg}$ of aflibercept every 4 weeks $(2 q 4)$ or every 8 weeks (2q8) after five initial monthly doses was associated with significantly better functional and anatomical outcomes as compared with laser photocoagulation. These favorable outcomes were sustained through weeks 100 and $148 .{ }^{26}$ In the Protocol T study, aflibercept was significantly superior to ranibizumab and bevacizumab in the subset of patients with worse vision (Early Treatment Diabetic Retinopathy Study [ETDRS] letter score $<69$, equivalent to $20 / 50$ or worse) at baseline. ${ }^{27}$ However, outside the framework of clinical trials, there is little evidence of real-life outcomes in patients with DME. Different studies have shown that converting unresponsive patients or those with persistent DME from bevacizumab or ranibizumab to aflibercept results in anatomical and vision improvements, ${ }^{28-33}$ but data in patients with treatment-naïve DME are lacking.

The purpose of the present study was to evaluate the effectiveness and safety profile of intravitreal aflibercept in the treatment-naïve patients with DME in daily clinical practice.

\section{Methods Study design and patients}

This was a prospective, single-center, 12-month observational study carried out in a 240-bed district general hospital in Plasencia, Spain. The study protocol was approved by the Ethics Committee of Hospital Virgen del Puerto of Plasencia, Cáceres (Spain), and the study was conducted in accordance with the principles of the Declaration of Helsinki for the protection of human subjects. Written informed consent was obtained from all individual participants included in the study.

Patients of both sexes, aged $>18$ years, were enrolled during routine ophthalmologic appointments between March 2015 and March 2016. All patients who were treatment-naïve of anti-VEGF agents with clinically significant DME were eligible for enrollment. Clinically significant DME was diagnosed on clinical examination and confirmed by spectraldomain optical coherence tomography (SD-OCT) (Cirrus HD-OCT; Carl Zeiss Meditec, Dublin, CA, USA). Clinically significant DME was measured within $500 \mu \mathrm{m}$ of the center of the macula and according to the ETDRS ${ }^{34}$ was defined upon slit lamp biomicroscopy as 1) thickening of the retina at or within $500 \mu \mathrm{m}$ of the center of the macula; or 2) hard exudate at or within $500 \mu \mathrm{m}$ of the center of the macula associated with thickening of adjacent retina; or 3) a zone of retinal thickening 1 disc area or larger, any part of which is within 1 disc diameter of the center of the macula.

To be included in the study, patients should be able to receive intravitreal treatment with aflibercept and not have undergone major ocular surgical procedures in the 4 months prior to the study or anticipated to undergo within the next 6 months after enrollment. Patients unable to participate in the study according to criteria of the ophthalmologist in charge and those who refused to sign the written informed consent were excluded. The study was carried out under conditions of daily practice. The duration of the study was 12 months.

\section{Study procedures}

All patients were treated with intravitreal aflibercept according to the Summary of Product Characteristics (SPC) and the treating physician's normal clinical practice. Patients received five loading doses of aflibercept injections $(2 \mathrm{mg} / 0.05 \mathrm{~mL}$, Eylea $^{\circledR}$; Bayer Hispania, S.L., Barcelona, Spain) every 4 weeks and then a fixed dose every 8 weeks. Injection of intravitreal aflibercept was performed as an outpatient procedure using topical anesthesia with lidocaine eye drops and strict sterile conditions. After topical anesthesia, the ocular surface and the lid were disinfected with povidone-iodine. 
Intravitreal injection of aflibercept was carried out using a 30 -gauge needle at $3.5 \mathrm{~mm}$ posterior to the limbus. The injection site was compressed by cotton swab to avoid reflux, and the fundus was examined to rule out any complication and to check perfusion of the retinal artery.

Patients were visited at the outpatient clinics of the Service of Ophthalmology at baseline, after the five loading doses, and at 2-month intervals thereafter, with a total of five visits (visit 1 baseline, visit 2 at 5 months, visit 3 at 7 months, visit 4 at 9 months, and visit 5 at 12 months). At each visit, a complete ophthalmologic examination was performed, which included slit lamp examination, best corrected visual acuity (BCVA) using an ETDRS optotype at $2 \mathrm{~m}$ distance from the observer, funduscopy, central subfield macular thickness, and vitreomacular adhesion (VMA) and/or vitreomacular traction (VMT) by SD-OCT. The presence of subretinal fluid and/or subfoveal or extrafoveal retinal cysts was assessed at the final visit. Also, serum glycosylated hemoglobin (HbAlc) levels were measured at baseline and at 6 and 12 months. Laser photocoagulation was included in the protocol in case of decrease in BCVA during the study period.

\section{Study outcomes}

The mean changes in BCVA (ETDRS letters) from baseline to the final study visit at 12 months were the primary outcomes of the study. Secondary outcomes were changes in central macular thickness from baseline to the final study visit at 12 months, changes in BCVA and central macular thickness after the five loading doses at each study visit, presence of VMA/VMT, changes in serum HbA1c levels at 6 and 12 months as compared with baseline, and the proportion of patients with subretinal fluid and/or retinal cysts at 12 months.

\section{Statistical analysis}

Categorical data are expressed as frequencies and percentages, and continuous data as mean and SD. Continuous variables during the different time points throughout the study period were analyzed with the Student's $t$-test for paired samples. Statistical significance was set at $P<0.05$. The Statistical Analysis Systems (SAS Institute, Cary, NC, USA) version 9.4 was used for analysis.

\section{Results}

A total of 29 eyes from 15 patients were included in the study and were followed for 12 months. There were 11 men and 4 women, with a mean age of 55.6 years (range 36-76 years). Baseline BCVA ranged between 20 and 72 ETDRS letters.
At baseline, VMA/VMT was documented in nine eyes $(31.9 \%)$ associated with epiretinal membrane (ERM) in five. In these five patients, the diagnosis of incipient ERM was established by OCT. At the end of the follow-up period, VMA/VMT was still present in three eyes (10.3\%), with ERM in one. Also, subretinal fluid and/or extrafoveal cysts were present in all eyes at baseline. At the final visit at month 12 , subretinal fluid was detected in 18 eyes (62.1\%) and extrafoveal retinal cysts in $5(17.2 \%)$.

In relation to the primary outcome of the study (Figure 1), the mean BCVA improved significantly as compared with baseline at 12 months of treatment (47.3 [14.2] vs 62.2 [13.9] ETDRS letters, $P<0.001)$. The mean difference was 14.9 (6.5) (95\% CI 12.5 to 17.4). Significant improvement in BCVA was already observed at visit 2 after the loading doses of aflibercept (47.3 [14.2] vs 60.3 [14.0] ETDRS letters, $P<0.001)$, with a mean difference of 13.0 (5.9) (95\% CI 10.7 to 15.2). Comparisons of BCVA between baseline and results at visits 3,4 , and 5 were also statistically significant $(P<0.001)$. However, changes in BCVA between follow-up visits were not significant (Table 1). At 12 months, gains in ETDRS letters were documented in all eyes (100\%), with gains $\geq 10$ letters in $89.6 \%(n=26), \geq 15$ letters in $65.5 \%$ $(\mathrm{n}=19)$, and $\geq 20$ letters in $6.9 \%(\mathrm{n}=2)$. There were no cases of vision loss.

Treatment with intravitreal aflibercept was associated with a significant reduction in central macular thickness from a mean of 460.5 (11.8) $\mu \mathrm{m}$ at baseline to 229.0 (43.8) $\mu \mathrm{m}$ at 12 months $(P<0.001)$. The mean difference was -231.5 (126.6) $\mu \mathrm{m}$ (95\% CI -279.7 to -183.3$)$. Significant reductions in central macular thickness were already observed at visit 2 after the loading doses of aflibercept (Figure 2). Comparisons of central macular thickness between baseline

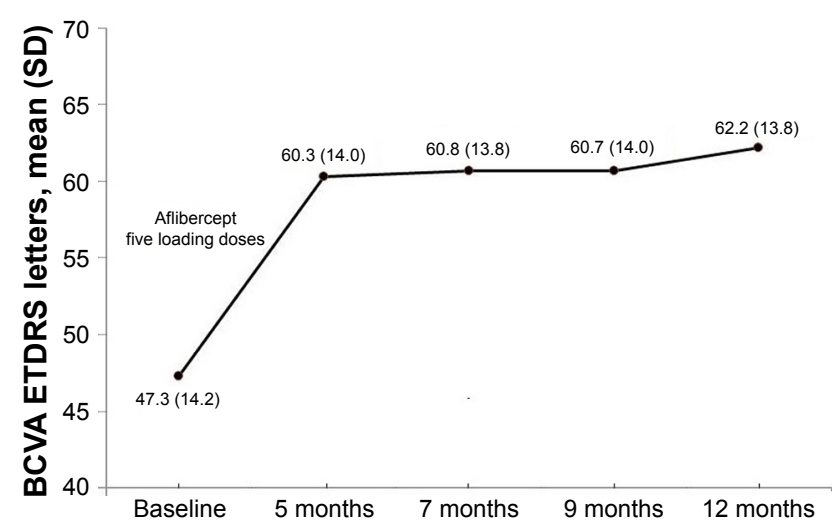

Figure I Changes in BCVA ETDRS letters during the study period. Abbreviations: BCVA, best-corrected visual acuity; ETDRS, Early Treatment Diabetic Retinopathy Study. 
Table I Changes in BCVA in 29 study eyes from 15 patients with DME

\begin{tabular}{|c|c|c|c|c|c|}
\hline \multirow[t]{3}{*}{ Time points } & \multicolumn{4}{|c|}{ BCVA ETDRS letters } & \multirow[t]{3}{*}{$P$-value } \\
\hline & \multirow[t]{2}{*}{ Mean (SD) } & \multirow[t]{2}{*}{$95 \% \mathrm{Cl}$} & \multicolumn{2}{|c|}{ Crude difference } & \\
\hline & & & Mean (SD) & $95 \% \mathrm{Cl}$ & \\
\hline Baseline & $47.3(14.2)$ & 41.9 to 52.7 & & & \\
\hline \multicolumn{6}{|l|}{ Intravitreal aflibercept } \\
\hline Visit 2 (5 months) & $60.3(14.0)$ & 55.9 to 65.7 & $13.0(5.9)$ & 10.7 to 15.2 & $<0.001$ \\
\hline Visit 3 (7 months) & $60.8(13.8)$ & 55.5 to 66.0 & I $3.4(6.8)$ & 10.9 to 16.0 & $<0.001$ \\
\hline Visit 4 (9 months) & $60.7(14.0)$ & 55.4 to 66.0 & $13.4(7.0)$ & 10.7 to 6.1 & $<0.001$ \\
\hline Visit 5 (I2 months) & $62.2(13.8)$ & 57.0 to 67.5 & $14.9(6.5)$ & 12.5 to 17.4 & $<0.001$ \\
\hline Visit 3 vs visit 2 & & & $0.48(4.9)$ & -1.3 to 2.4 & 0.602 \\
\hline Visit 4 vs visit 3 & & & $-0.03(6.1)$ & -2.4 to 2.3 & 0.975 \\
\hline Visit 5 vs visit 4 & & & $1.5(4.2)$ & -0.09 to 3.1 & 0.063 \\
\hline
\end{tabular}

Abbreviations: BCVA, best-corrected visual acuity; DME, diabetic macular edema; ETDRS, Early Treatment Diabetic Retinopathy Study.

and results at visits 3,4 , and 5 were also statistically significant $(P<0.001)$. Moreover, changes in central macular thickness between visits 3 to 4 and 4 to 5 were also statistically significant (Table 2 ).

In relation to serum levels of $\mathrm{HbA} 1 \mathrm{c}$, mean (SD) baseline levels were $8.0 \%(0.5)$ as compared to $7.9 \%(0.3)$ at 6 months $(P=0.247)$ and $7.9 \%(0.5)$ at 12 months $(P=0.280)$.

Ocular or systemic adverse events related to intravitreal treatment with aflibercept were not recorded. None of the patients received other treatment (eg, cataract surgery and refractive surgery) during the 12-month study period.

\section{Discussion}

The present prospective study carried out in daily clinical practice confirms the effectiveness of intravitreal aflibercept for the treatment of patients with clinically significant DME and naïve to anti-VEGF treatment. Adverse events related to intravitreal aflibercept were not recorded. Our data are consistent with optimal vision gains and anatomic improvement previously reported in the large double-masked, randomized Phase III clinical trials VISTA-DME and VIVID-DME. ${ }^{24,25}$

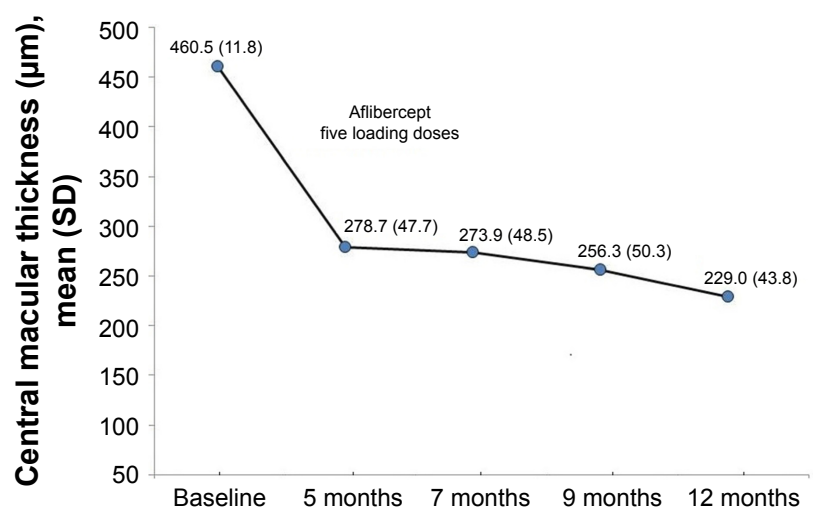

Figure 2 Changes in central macular thickness from baseline to 12 months after treatment with intravitreal aflibercept.
In these trials, patients were randomized to intravitreal aflibercept injection $2 \mathrm{mg}$ every 4 weeks (2q4; n=290), $2 \mathrm{mg}$ every 8 weeks $(2 \mathrm{q} 8 ; \mathrm{n}=286)$ after five initial monthly doses, or macular laser photocoagulation $(n=286)$. The primary efficacy end point was the change from baseline in BCVA in ETDRS letters at week 52. Treatment with aflibercept was associated with gains in ETDRS letters of 12.5, 10.7, and 0.2 in the $2 \mathrm{q} 4,2 \mathrm{q} 8$, and laser groups, respectively. Results obtained in our study with a mean difference of 13.0 ETDRS letters are even more favorable than mean BCVA gains reported in these trials. Also, in relation to anatomic improvement, reductions in central macular thickness with a mean difference of $231.5 \mu \mathrm{m}$ found in the present clinical series are greater than 185.9 and $195.0 \mu \mathrm{m}$, and 183.1 and $192.4 \mu \mathrm{m}$ reported for the $2 \mathrm{q} 4$ and $2 \mathrm{q} 8$ regimens of aflibercept, respectively, in the two randomized trials.

There are limited data on the effect of aflibercept for the management of DME in the real-world setting, and the majority of previously published studies have evaluated the efficacy of aflibercept in patients with DME unresponsive to previous anti-VEGF therapy. ${ }^{28-33}$ Recently, a randomized prospective study conducted in daily practice in 70 eyes with DME divided into two groups of 35 eyes each, compared intravitreal aflibercept and ranibizumab after a follow-up of 12 months. ${ }^{35}$ At 12 months, similar efficacy for both drugs in the treatment of DME in eyes with moderate visual loss was demonstrated, but with less number of drug reinjection and treatment burden for aflibercept. In this study, it is not explicitly mentioned if patients were naïve to antiVEGF treatment nor if they had clinically significant DME; only three loading doses of aflibercept were administered unlike five loading doses recommended in the SPC; and anti-VEGF was reinjected if the macular edema was persistent or if the visual acuity or central macular thickness worsened, which is different from the regimen of fixed 
Table 2 Changes in central macular thickness in 29 study eyes from 15 patients with DME

\begin{tabular}{|c|c|c|c|c|c|}
\hline \multirow[t]{3}{*}{ Time points } & \multicolumn{4}{|c|}{ Central macular thickness $(\mu \mathrm{m})$} & \multirow[t]{3}{*}{$P$-value } \\
\hline & \multirow[t]{2}{*}{ Mean (SD) } & \multirow[t]{2}{*}{$95 \% \mathrm{Cl}$} & \multicolumn{2}{|c|}{ Crude difference } & \\
\hline & & & Mean (SD) & $95 \% \mathrm{Cl}$ & \\
\hline Baseline & 460.5 (III.8) & 417.9 to 503.0 & & & \\
\hline \multicolumn{6}{|l|}{ Intravitreal aflibercept } \\
\hline Visit 2 (5 months) & $278.7(47.7)$ & 260.6 to 296.9 & $-181.8(120.5)$ & -227.6 to -135.9 & $<0.001$ \\
\hline Visit 3 (7 months) & $273.9(48.5)$ & 255.4 to 292.4 & $-186.6(108.6)$ & -227.9 to -145.3 & $<0.001$ \\
\hline Visit 4 (9 months) & $256.3(50.3)$ & 237.2 to 275.5 & $-204.1(124.8)$ & -251.6 to -156.7 & $<0.001$ \\
\hline Visit 5 (I2 months) & $229.0(43.8)$ & 212.3 to 245.6 & $-231.5(\mid 26.6)$ & -279.7 to -183.3 & $<0.001$ \\
\hline Visit 3 vs visit 2 & & & $-4.8(45.1)$ & -22.0 to 12.3 & 0.569 \\
\hline Visit 4 vs visit 3 & & & $-17.5(34.4)$ & -30.6 to -4.5 & 0.010 \\
\hline Visit 5 vs visit 4 & & & $-27.4(35.0)$ & -40.7 to -14.1 & $0.00 \mathrm{I}$ \\
\hline
\end{tabular}

Abbreviation: DME, diabetic macular edema.

doses at 2-month intervals used in our study. However, this randomized study also provides evidence of the effectiveness of aflibercept in patients with DME who attended the routine clinical setting.

In this study, we report the prospective data collected in the real-world setting from 15 patients (29 eyes) with clinically significant DME who were naïve for anti-VEGF therapy, followed for 12 months, in whom aflibercept was indicated as a first-line therapy and administered according to technical specifications of the drug. Aflibercept as the first choice of treatment in these patients resulted in a prompt and substantial functional and anatomic improvement, with statistically significant differences as compared with pretreatment values at 5 months of treatment (after the loading dose of the drug). All patients maintained improved vision at 12 months. In addition, further significant reductions in central foveal thickness along the study period were observed. Also, resolution of VMA/VMT was found in $66.7 \%$ of patients.

Limitations of the study include the small sample size and the open-label design where selection bias cannot be excluded, although recommended criteria for the definition of clinically significant DME were used. However, under routine daily practice circumstances, the present findings show that intravitreal treatment with aflibercept was useful as a first-line therapy in patients with treatment-naïve DME, with sustained BCVA improvement and reduction in central macular thickness.

\section{Acknowledgment}

The authors thank Marta Pulido, MD, for editing the manuscript and editorial assistance.

\section{Disclosure}

The authors report no conflicts of interest in this work.

\section{References}

1. Cohen SR, Gardner TW. Diabetic retinopathy and diabetic macular edema. Dev Ophthalmol. 2016;55:137-146.

2. Cheung N, Mitchell P, Wong TY. Diabetic retinopathy. Lancet. 2010; 376(9735):124-136.

3. Ding J, Wont TY. Current epidemiology of diabetic retinopathy and diabetic macular edema. Curr Diab Rep. 2012;12(4):346-354.

4. Lee R, Wong TY, Sabanayagam C. Epidemiology of diabetic retinopathy, diabetic macular edema and related vision loss. Eye Vis (Lond). 2015;2:17.

5. Executive Summary. IFD Diabetes Atlas, 7th edition. Available from: http://www.diabetesatlas.org/. Accessed July 9, 2017.

6. Bandello F, Cicinelli MV, Parodi MB. Anti-VEGF molecules for the management of diabetic macular edema. Curr Pharm Des. 2015; 21(32):4731-4737.

7. Fogli S, Mogavero S, Egan CG, Del Re M, Danesi R. Pathophysiology and pharmacological targets of VEGF in diabetic macular edema. Pharmacol Res. 2016;103:149-157.

8. Stewart MW. The expanding role of vascular endothelial growth factor inhibitors in ophthalmology. Mayo Clin Proc. 2012;87(1):77-88.

9. Simó R, Sundstrom JM, Antonetti DA. Ocular Anti-VEGF therapy for diabetic retinopathy: the role of VEGF in the pathogenesis of diabetic retinopathy. Diabetes Care. 2014;37(4):893-899.

10. Ajlan RS, Silva PS, Sun JK. Vascular endothelial growth factor and diabetic retinal disease. Semin Ophthalmol. 2016;31(1-2):40-48.

11. Virgili G, Parravano M, Menchini F, Brunetti M. Antiangiogenic therapy with anti-vascular endothelial growth factor modalities for diabetic macular edema. Cochrane Database Syst Rev. 2012;12:CD007419.

12. Bandello F, Berchicci L, La Spina C, Battaglia Parodi M, Iacono P. Evidence for anti-VEGF treatment of diabetic macular edema. Ophthalmic Res. 2012;48(Suppl 1):16-20.

13. Goyal S, Lavalley M, Subramanian ML. Meta-analysis and review on the effect of bevacizumab in diabetic macular edema. Graefes Arch Clin Exp Ophthalmol. 2011;249(1):15-27.

14. Yilmaz T, Cordero-Coma M, Gallagher MJ, Teasley LA. Systematic review of intravitreal bevacizumab injection for treatment of primary diabetic macular edema. Acta Ophthalmol. 2011;89(8):709-717.

15. Dervenis N, Mikropoulou AM, Tranos P, Dervenis P. Ranibizumab in the treatment of diabetic macular edema: a review of the current status, unmet needs, and emerging challenges. Adv Ther. 2017;34(6): $1270-1282$.

16. Abouammoh MA. Ranibizumab injection for diabetic macular edema: meta-analysis of systemic safety and systematic review. Can J Ophthalmol. 2013;48(4):317-323.

17. Chang AA, Hong T, Ewe SY, Bahrami B, Broadhead GK. The role of aflibercept in the management of diabetic macular edema. Drug Des Devel Ther. 2015;9:4389-4396. 
18. Harkins KA, Haschke M, Do DV. Aflibercept for the treatment of diabetic macular edema. Immunotherapy. 2016;8(5):503-510.

19. Keating GM. Aflibercept: a review of its use in diabetic macular edema. Drugs. 2015;75(10):1153-1160.

20. Moradi A, Sepah YJ, Sadiq MA, et al. Vascular endothelial growth factor trap-eye (Aflibercept) for the management of diabetic macular edema. World J Diabetes. 2013;4(6):303-309.

21. Korobelnik JF, Kleijnen J, Lang SH, et al. Systematic review and mixed treatment comparison of intravitreal aflibercept with other therapies for diabetic macular edema (DME). BMC Ophthalmol. 2015;15:52.

22. Deissler HL, Lang GK, Lang GE. Capacity of aflibercept to counteract VEGF-stimulated abnormal behavior of retinal microvascular endothelial cells. Exp Eye Res. 2014;122:20-31.

23. Stewart MW, Rosenfeld PJ. Predicted biological activity of intravitreal VEGF Trap. Br J Ophthalmol. 2008;92(5):667-668.

24. Korobelnik JF, Do DV, Schmidt-Erfurth U, et al. Intravitreal aflibercept for diabetic macular edema. Ophthalmology. 2014;121(11): 2247-2254.

25. Brown DM, Schmidt-Erfurth U, Do DV, et al. Intravitreal aflibercept for diabetic macular edema: 100-week results from the VISTA and VIVID studies. Ophthalmology. 2015;122(10):2044-2052.

26. Heier JS, Korobelnik JF, Brown DM, et al. Intravitreal aflibercept for diabetic macular edema: 148-week results from the VISTA and VIVID studies. Ophthalmology. 2016;123(11):2376-2385.

27. The Diabetic Retinopathy Clinical Research Network. Aflibercept, bevacizumab, or ranibizumab for diabetic macular edema. $N$ Engl $J$ Med. 2015;372:1193-1203.

28. Konidaris VE, Tsaousis KT, Al-Hubeshy Z, Pieri K, Deane J, Empeslidis T. Clinical real-world results of switching treatment from ranibizumab to aflibercept in patients with diabetic macular edema. Eye (Lond). 2017;31(11):1629-1630.
29. Bahrami B, Hong T, Zhu M, Schlub TE, Chang A. Switching therapy from bevacizumab to aflibercept for the management of persistent diabetic macular edema. Graefes Arch Clin Exp Ophthalmol. 2017; 255(6):1133-1140.

30. Călugăru D, Călugăru M. Switching therapy from bevacizumab to aflibercept for the management of persistent diabetic macular edema. Graefes Arch Clin Exp Ophthalmol. 2017;255(7):1451-1452.

31. Lim LS, Ng WY, Mathur R, et al. Conversion to aflibercept for diabetic macular edema unresponsive to ranibizumab or bevacizumab. Clin Ophthalmol. 2015;9:1715-1718.

32. Wood EH, Karth PA, Moshfeghi DM, Leng T. Short-term outcomes of aflibercept therapy for diabetic macular edema in patients with incomplete response to ranibizumab and/or bevacizumab. Ophthalmic Surg Lasers Imaging Retina. 2015;46(9):950-954.

33. Mira F, Paulo M, Henriques F, Figueira J. Switch to aflibercept in diabetic macular edema patients unresponsive to previous anti-VEGF therapy. J Ophthalmol. 2017;2017:5632634.

34. Early Treatment Diabetic Retinopathy Study Research Group. Grading diabetic retinopathy from stereoscopic color fundus photographs an extension of the modified Airlie House classification. ETDRS report number 10. Ophthalmology. 1991;98(5 Suppl):786-806.

35. Fouda SM, Bahgat AM. Intravitreal aflibercept versus intravitreal ranibizumab for the treatment of diabetic macular edema. Clin Ophthalmol. 2017;11:567-571.
Clinical Ophthalmology

\section{Publish your work in this journal}

Clinical Ophthalmology is an international, peer-reviewed journal covering all subspecialties within ophthalmology. Key topics include: Optometry; Visual science; Pharmacology and drug therapy in eye diseases; Basic Sciences; Primary and Secondary eye care; Patient Safety and Quality of Care Improvements. This journal is indexed on Submit your manuscript here: http://www.dovepress.com/clinical-ophthalmology-journal

\section{Dovepress}

PubMed Central and CAS, and is the official journal of The Society of Clinical Ophthalmology (SCO). The manuscript management system is completely online and includes a very quick and fair peer-review system, which is all easy to use. Visit http://www.dovepress.com/ testimonials.php to read real quotes from published authors. 